\title{
Extranasopharyngeal Angiofibroma Originating in the Inferior Turbinate: A Distinct Clinical Entity at an Unusual Site
}

\author{
Marco Antonio Ferraz de Barros Baptista ${ }^{1,2}$ Fábio de Rezende Pinna ${ }^{2}$ Richard Louis Voegels ${ }^{2}$ \\ ${ }^{1}$ Department of Otolaryngology, Hospital das Clínicas da Faculdade de \\ Medicina da Universidade de São Paulo, HCFMUSP, São Paulo, \\ SP, Brazil \\ 2 Department of Otolaryngology, Faculdade de Medicina da \\ Universidade de São Paulo, São Paulo, Brazil \\ Address for correspondence Marco Antonio Ferraz de Barros Baptista, \\ Departamento de Otorrinolaringologia, Hospital das Clínicas, \\ Universidade de São Paulo, Av. Dr. Enéas de Carvalho Aguiar, 255 - 6 \\ andar / sala 6167, São Paulo, SP 05403-000, Brazil \\ (e-mail: medicinamarco@gmail.com).
}

Int Arch Otorhinolaryngol 2014;18:403-405.

\begin{abstract}
Introduction The extranasopharyngeal angiofibroma is histologically similar to juvenile nasopharyngeal angiofibroma, differing from the latter in clinical and epidemiologic characteristics.

Objectives We present a case of extranasopharyngeal angiofibroma originating in the inferior turbinate.

Resumed Report The patient was a girl, 8 years and 6 months of age, who had constant bilateral nasal obstruction and recurrent epistaxis for 6 months, worse on the right side, with hyposmia and snoring. Nasal endoscopy showed a reddish lesion, smooth, friable, and nonulcerated. Computed tomography showed a lesion with soft tissue density in the right nasal cavity. We used an endoscopic approach and found the lesion inserted in the right inferior turbinate. We did a subperiosteal dissection and

Keywords

- angiofibroma

- differential diagnosis

- inferior turbinate excision with a partial turbinectomy with a resection margin of $0.5 \mathrm{~cm}$. Histopathology reported it to be an extranasopharyngeal angiofibroma.

Conclusion Although rare, extranasopharyngeal angiofibroma should be considered in the diagnosis of vascular tumors of the head and neck.
\end{abstract}

\section{Introduction}

The extranasopharyngeal angiofibroma (ENPA) is histologically similar to juvenile nasopharyngeal angiofibroma (JNA), differing from the latter in clinical and epidemiologic characteristics. ${ }^{1-3}$ Prevalence, gender, age, affected site, pathogenesis, clinical and computed tomography characteristics, and recurrence are completely different, ${ }^{1-3}$ which leads some authors to classify the ENPA as a disease different from the JNA. ${ }^{1}$

There are fewer than a hundred cases of ENPA described in the literature, and the maxillary sinus is the most frequently affected site, followed by the ethmoid; the entity is rare in the nasal septum and inferior turbinates. ${ }^{1-3}$ The objective of this study is to report a case of ENPA with a rare presentation in the inferior turbinate.

\section{Review of Literature with Differential Diagnosis}

Angiofibromas that originate in or are localized in an area other than the nasopharynx are called extranasopharyngeal angiofibromas or atypical angiofibromas. ${ }^{2,3}$

The JNA is the most common benign neoplasm of the nasopharynx, despite representing less than $0.05 \%$ of tumors of the head and neck. ${ }^{1,2,4,5}$ It affects almost exclusively males between 12 and 14 years of age. , $^{1,4,5}$ But the ENPA is even more unusual; it is more common in females between 17 and 22 years, received

March 25, 2014

accepted after revision

July 6, 2014

published online

August 25, 2014
DOI http://dx.doi.org/

10.1055/s-0034-1387811. ISSN $1809-9777$.
Copyright $\odot 2014$ by Thieme Publicações License terms Ltda, Rio de Janeiro, Brazil

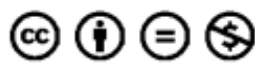


and its most common site is the maxillary sinus, followed by the ethmoid. It is very rare in the nasal septum and inferior turbinates. $^{1-3}$ Angiofibromas that originate in the inferior turbinate are very rare; to the best of our knowledge, only nine cases of ENPA in nasal cavity have been previously reported in the English language literature. In seven cases, the tumor arose from the nasal septum, and only two from inferior turbinate. ${ }^{6-10}$

The origin of the JNA is at the top of the sphenopalatine foramen, ${ }^{1,2,5}$ and its etiology is controversial. ${ }^{4}$ ENPA's etiology is associated with a migration error of the fascia basalis, ${ }^{1}$ justifying its presence in varied locations. ${ }^{2}$

The initial growth of the JNA follows a well-defined pattern in the nasal cavity, nasopharynx, and pterygopalatine fossa, ${ }^{4}$ leading to the triad of nasal obstruction, recurrent epistaxis, and nasopharyngeal tumor. ${ }^{1,2,5}$ The JNA has characteristic radiologic signs: Holman-Miller (anteriorization of the posterior wall of the maxillary sinus) and enlargement of the sphenopalatine foramen and pterygopalatine fossa. $1,2,4,5$

Histologically, the ENPA is similar to the JNA, with connective tissue stroma and a matrix of dilated vessels without a muscular layer. ${ }^{2,3,5}$ As for differential diagnosis, we have the hemangioma and the hemangiopericitoma. ${ }^{3}$ Although the JNA can be suspected based on known clinical and computed tomography (CT) chracteristics, ${ }^{2,4,5}$ histopathologic examination is essential to confirm the ENPA diagnosis. ${ }^{1}$

Treatment is surgical in both diseases. ${ }^{2}$ Although the ENPA is nurtured by the maxillary artery ${ }^{4}$ (just like the JNA), it may not cause excessive intraoperative bleeding due to the predominance of fibrous stroma, unlike the JNA. ${ }^{1,2}$ Although benign, the JNA is locally aggressive, with recurrence rates of 6 to $27.5 \%^{2}$ due to incomplete tumor removal. ${ }^{5}$

\section{Case Report}

The patient was a girl, 8 years and 6 months of age, who had constant bilateral nasal obstruction and recurrent epistaxis for 6 months, worse on the right side, with hyposmia and snoring. Nasal endoscopy showed a reddish lesion, smooth,

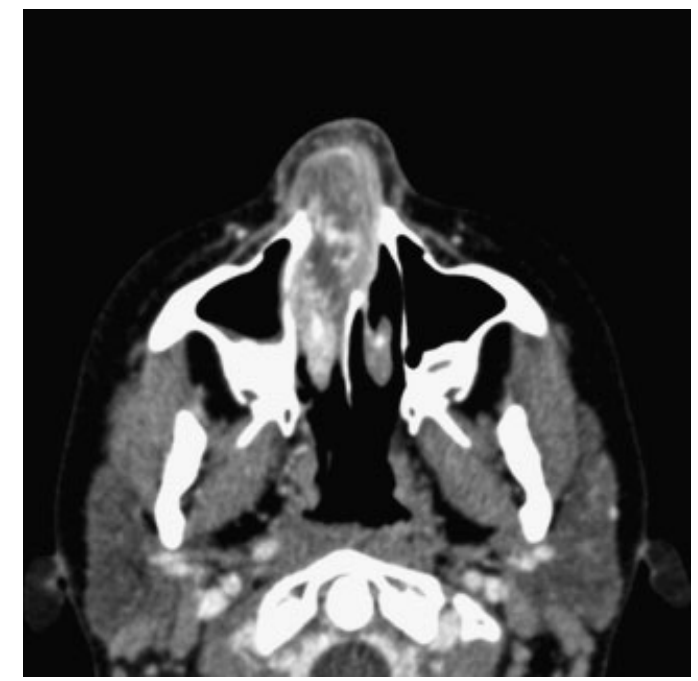

Fig. 1 Preoperative axial computed tomography scan showing the tumor in the right nasal cavity with contrast enhancement.

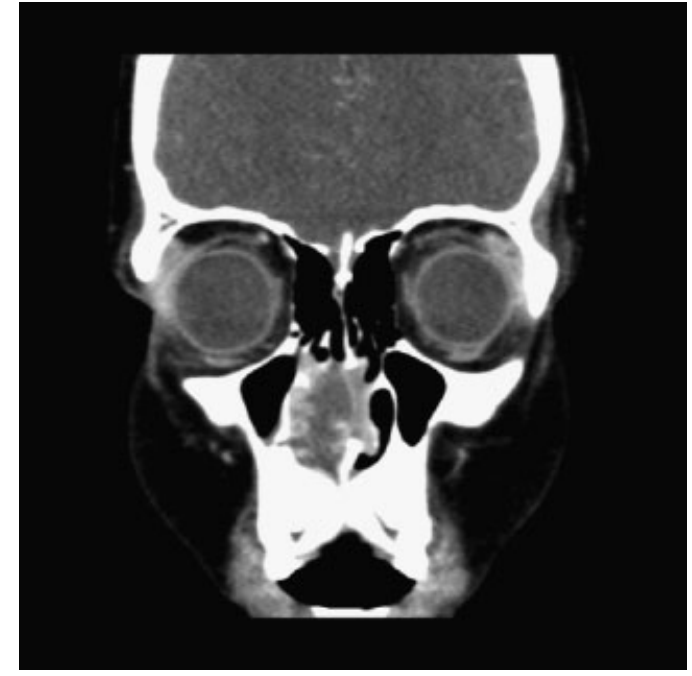

Fig. 2 Preoperative coronal computed tomography scan showing the tumor in the right nasal cavity with contrast enhancement.

friable, and nonulcerated. The nasal mass obstructed the right nasal vestibule. The left nasal cavity and nasopharynx were normal, and no cervical lymphadenopathy was present. Middle meatuses and sphenoethmoidal recesses were free in the left side. CT showed a lesion with soft tissue density in the right nasal cavity (-Figs. 1, 2, and 3). We suspected a vascular tumor and decided to remove the mass.

We used an endoscopic approach, identifying the lesion inserted in the right inferior turbinate, doing a subperiosteal dissection and excision with a partial turbinectomy with a resection margin of $0.5 \mathrm{~cm}$. We did not perform preoperative embolization because the tumor was at an accessible location, and we expected that the bleeding could be easily controlled. However, the patient experienced excessive intraoperative bleeding ( $800 \mathrm{~mL}$ in 5 minutes). On gross inspection, the tumor was a $6 \times 4$-cm rubbery mass with smooth margins (-Fig. 4). On histopathology, it was composed of fibrous stroma and numerous thin-walled blood vessels (-Fig. 5). Based on the

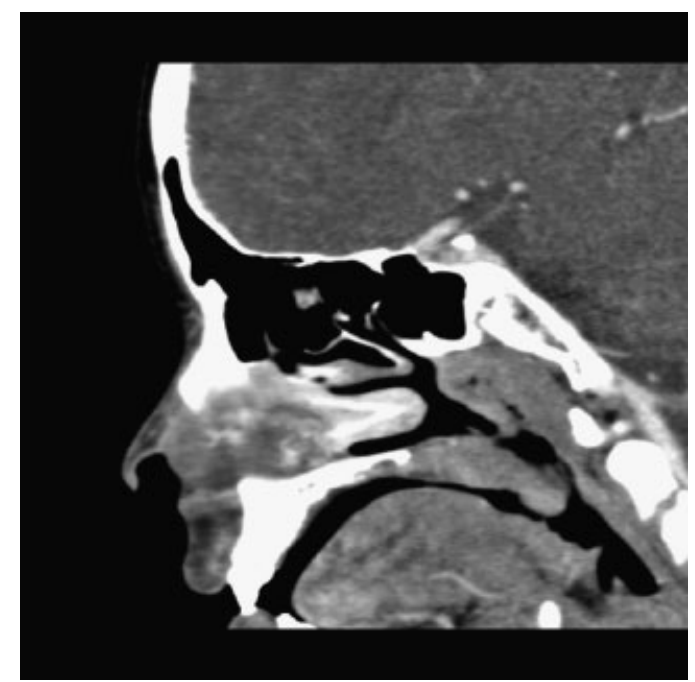

Fig. 3 Preoperative sagittal computed tomography scan showing the tumor in the right nasal cavity with contrast enhancement. 


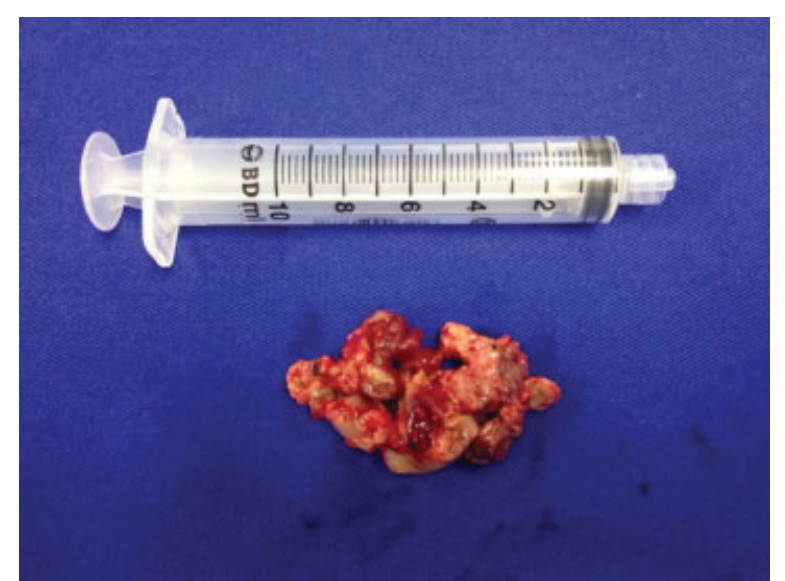

Fig. 4 Extranasopharyngeal angiofibroma after endoscopic resection.

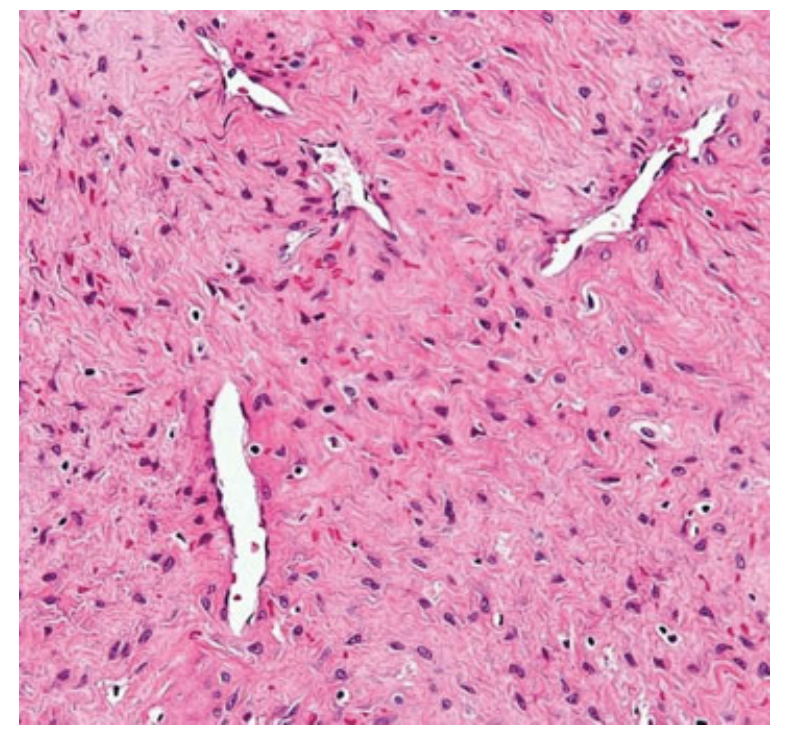

Fig. 5 Histologic examination: abundant fibrous component with thin-walled vascular structures (hematoxylin-eosin staining, original magnification $\times 400)$.

histopathologic findings, the tumor was diagnosed as an angiofibroma. The histopathologic findings were revised and the ENPA diagnosis was confirmed by immunohistochemical findings. The patient's postoperative course was uneventful, and she showed no further symptoms. Over postoperative follow-up of 6 months, no recurrence was noted.

\section{Discussion}

ENPAs are usually found in the maxillary sinus. ${ }^{6}$ Angiofibromas that originate in the inferior turbinate are very rare; to the best of our knowledge, only nine cases of ENPA in the nasal cavity have been previously reported in the English language literature. In seven cases, the tumor arose from the nasal septum and only two arose from the inferior turbinate. ${ }^{6-10}$

The ENPA can evolve with a variety of symptoms and radiologic signs, depending on its site. ${ }^{1,2}$ Our patient reported nasal obstruction due to a rare location in the right inferior turbinate.
The ENPA usually does not recur because its extrapharyngeal location facilitates total ressection. ${ }^{1-3}$ Our patient did not complain of postoperative epistaxis, but had excessive intraoperative bleeding. She is now 9 years old; there has been no recurrence in 6 months.

Our patient had age and location different from most ENPAs, confirming the rarity of this case.

Therefore, although histologically similar, the ENPA and the JNA may be considered different diseases, due to totally different pathogenesis, epidemiology, and clinical and tomographic presentations. ${ }^{1}$

This case challenges all the pathogenetic and evolutionary characteristics of JNA and ENPA. Cavernous hemangioma, hemangiopericytoma, or pyogenic granuloma is more plausible and this is more likely with the radiologic findings. Therefore, the histopathology was revised and the ENPA diagnosis was confirmed.

\section{Final Comments}

Although rare, ENPA should be considered in the diagnosis of vascular tumors of the head and neck. ENPA's clinical and epidemiologic characteristics are different from those of JNA.

In conclusion, ENPA differs significantly from JNA regarding clinical and radiologic presentations. ENPAs lack typical clinical and radiologic features as they develop in all age groups and in females. They arise from various sites, may be less vascularized, and produce a variety of symptoms depending on the point of origin.

\section{References}

1 Windfuhr JP, Remmert S. Extranasopharyngeal angiofibroma: etiology, incidence and management. Acta Otolaryngol 2004; 124(8):880-889

2 Szymańska A, Szymański M, Morshed K, Czekajska-Chehab E, Szczerbo-Trojanowska M. Extranasopharyngeal angiofibroma: clinical and radiological presentation. Eur Arch Otorhinolaryngol 2013;270(2):655-660

3 Garcia-Rodriguez L, Rudman K, Cogbill CH, Loehrl T, Poetker DM. Nasal septal angiofibroma, a subclass of extranasopharyngeal angiofibroma. Am J Otolaryngol 2012;33(4):473-476

4 Lund VJ, Stammberger H, Nicolai P, et al; European Rhinologic Society Advisory Board on Endoscopic Techniques in the Management of Nose, Paranasal Sinus and Skull Base Tumours. European position paper on endoscopic management of tumours of the nose, paranasal sinuses and skull base. Rhinol Suppl 2010;(22):1-143

5 Ricardo LAC, Tiago RSL, Fava AS. Nasopharyngeal angiofibroma: literature review. Rev Bras Otorrinolaringol 2003;69(3):394-403

6 Nomura K, Shimomura A, Awataguchi T, Murakami K, Kobayashi T. A case of angiofibroma originating from the inferior nasal turbinate. Auris Nasus Larynx 2006;33(2):191-193

7 Celik B, Erisen L, Saraydaroglu O, Coskun H. Atypical angiofibromas: a report of four cases. Int J Pediatr Otorhinolaryngol 2005; 69(3):415-421

8 Alvi A, Myssiorek D, Fuchs A. Extranasopharyngeal angiofibroma. J Otolaryngol 1996;25(5):346-348

9 Gaffney R, Hui Y, Vojvodich S, Forte V. Extranasopharyngeal angiofibroma of the inferior turbinate. Int J Pediatr Otorhinolaryngol 1997;40(2-3):177-180

10 Taggarshe D, Quraishi MS, Dugar JM. Inferior turbinate angiofibroma: an atypical presentation [correction of preservation]. Rhinology 2004;42(1):45-47 